Correction

\title{
Correction: Bennati et al. An Elastic Interface Model for the Delamination of Bending-Extension Coupled Laminates. Appl. Sci. 2019, 9, 3560
}

\section{Stefano Bennati $\mathbb{D}^{D}$, Paolo Fisicaro $\mathbb{D}^{D}$, Luca Taglialegne $\mathbb{D}^{\mathbb{D}}$ and Paolo S. Valvo *}

Department of Civil and Industrial Engineering, University of Pisa, Largo Lucio Lazzarino, I-56122 Pisa, Italy; s.bennati@ing.unipi.it (S.B.); p.fisicaro@ing.unipi.it (P.F.); 1.taglialegne@ing.unipi.it (L.T.)

* Correspondence: p.valvo@ing.unipi.it; Tel.: +39-050-2218223

Received: 18 October 2019; Accepted: 27 January 2020; Published: 3 March 2020

We, the authors, wish to make the following corrections to our paper [1].

Equations (26), (27), (28), and (41) were affected by some typos and should be substituted by the following ones (corrections are colored in red):

$$
\begin{aligned}
& u_{1}(x)=-\left(\mathrm{a}_{1}+\mathrm{b}_{1} h_{1}\right)\left[\frac{f_{5}}{\lambda_{5}^{2}} \exp \left(-\lambda_{5} x\right)+\frac{f_{6}}{\lambda_{6}^{2}} \exp \left(-\lambda_{6} x\right)\right]+\mathrm{b}_{1} \sum_{i=1}^{4} \frac{f_{i}}{\lambda_{i}^{3}} \exp \left(-\lambda_{i} x\right)+ \\
& -\left[\left(a_{1}+b_{1} h_{1}\right) f_{7}+b_{1} f_{10}\right] \frac{x^{2}}{2}-\left(a_{1} f_{8}+b_{1} f_{12}\right) x+f_{14} \\
& w_{1}(x)=\sum_{i=1}^{4}\left(\frac{\mathrm{d}_{1}}{\lambda_{i}^{2}}-\mathrm{c}_{1}\right) \frac{f_{i}}{\lambda_{i}^{2}} \exp \left(-\lambda_{i} x\right)-\left(\mathrm{d}_{1} h_{1}+\mathrm{b}_{1}\right)\left[\frac{f_{5}}{\lambda_{5}^{3}} \exp \left(-\lambda_{5} x\right)+\frac{f_{6}}{\lambda_{6}^{3}} \exp \left(-\lambda_{6} x\right)\right]+ \\
& +\left[\left(\mathrm{d}_{1} h_{1}+\mathrm{b}_{1}\right) f_{7}+\mathrm{d}_{1} f_{10}\right] \frac{x^{3}}{6}+\left(\mathrm{b}_{1} f_{8}+\mathrm{d}_{1} f_{12}\right) \frac{x^{2}}{2}-\left(f_{16}+\mathrm{c}_{1} f_{10}\right) x+f_{15}, \quad \text { and } \\
& \varphi_{1}(x)=\mathrm{d}_{1} \sum_{i=1}^{4} \frac{f_{i}}{\lambda_{i}^{3}} \exp \left(-\lambda_{i} x\right)-\left(\mathrm{d}_{1} h_{1}+\mathrm{b}_{1}\right)\left[\frac{f_{5}}{\lambda_{5}^{2}} \exp \left(-\lambda_{5} x\right)+\frac{f_{6}}{\lambda_{6}^{2}} \exp \left(-\lambda_{6} x\right)\right]+ \\
& -\left[\left(\mathrm{d}_{1} h_{1}+\mathrm{b}_{1}\right) f_{7}+\mathrm{d}_{1} f_{10}\right] \frac{x^{2}}{2}-\left(\mathrm{b}_{1} f_{8}+\mathrm{d}_{1} f_{12}\right) x+f_{16} \\
& u_{2}(x)=\left(\mathrm{a}_{2}-\mathrm{b}_{2} h_{2}\right)\left[\frac{f_{5}}{\lambda_{5}^{2}} \exp \left(-\lambda_{5} x\right)+\frac{f_{6}}{\lambda_{6}^{2}} \exp \left(-\lambda_{6} x\right)\right]-\mathrm{b}_{2} \sum_{i=1}^{4} \frac{f_{i}}{\lambda_{i}^{3}} \exp \left(-\lambda_{i} x\right)+ \\
& +\left[\left(\mathrm{a}_{2}-\mathrm{b}_{2} h_{2}\right) f_{7}+\mathrm{b}_{2} f_{11}\right] \frac{x^{2}}{2}+\left(\mathrm{a}_{2} f_{9}+\mathrm{b}_{2} f_{13}\right) x+f_{17} \\
& w_{2}(x)=-\sum_{i=1}^{4}\left(\frac{\mathrm{d}_{2}}{\lambda_{i}^{2}}-\mathrm{c}_{2}\right) \frac{f_{i}}{\lambda_{i}^{2}} \exp \left(-\lambda_{i} x\right)-\left(\mathrm{d}_{2} h_{2}-\mathrm{b}_{2}\right)\left[\frac{f_{5}}{\lambda_{5}^{3}} \exp \left(-\lambda_{5} x\right)+\frac{f_{6}}{\lambda_{6}^{3}} \exp \left(-\lambda_{6} x\right)\right] \\
& +\left[\left(\mathrm{d}_{2} h_{2}-\mathrm{b}_{2}\right) f_{7}-\mathrm{d}_{2} f_{11}\right] \frac{x^{3}}{6}-\left(\mathrm{b}_{2} f_{9}+\mathrm{d}_{2} f_{13}\right) \frac{x^{2}}{2}-\left(f_{19}-\mathrm{c}_{2} f_{11}\right) x+f_{18}, \quad \text { and } \\
& \varphi_{2}(x)=-\mathrm{d}_{2} \sum_{i=1}^{4} \frac{f_{i}}{\lambda_{i}^{3}} \exp \left(-\lambda_{i} x\right)-\left(\mathrm{d}_{2} h_{2}-\mathrm{b}_{2}\right)\left[\frac{f_{5}}{\lambda_{5}^{2}} \exp \left(-\lambda_{5} x\right)+\frac{f_{6}}{\lambda_{6}^{2}} \exp \left(-\lambda_{6} x\right)\right]+ \\
& -\left[\left(\mathrm{d}_{2} h_{2}-\mathrm{b}_{2}\right) f_{7}-\mathrm{d}_{2} f_{11}\right] \frac{x^{2}}{2}+\left(\mathrm{b}_{2} f_{9}+\mathrm{d}_{2} f_{13}\right) x+f_{19}
\end{aligned}
$$




$$
\begin{aligned}
& f_{10}=-\frac{\alpha_{3}}{B k_{x} \alpha_{4}} \mathrm{~d}_{2} f_{7}, \quad f_{11}=\frac{\alpha_{3}}{B k_{x} \alpha_{4}} \mathrm{~d}_{1} f_{7} \\
& f_{12}=-\frac{\left(\mathrm{a}_{1}+\mathrm{b}_{1} h_{1}\right) \mathrm{d}_{2}-\left(\mathrm{b}_{2}-\mathrm{d}_{2} h_{2}\right) \mathrm{b}_{1}}{\alpha_{4}} f_{8}-\frac{\mathrm{a}_{2} \mathrm{~d}_{2}-\mathrm{b}_{2}^{2}}{\alpha_{4}} f_{9} \\
& f_{13}=\frac{\mathrm{a}_{1} \mathrm{~d}_{1}-\mathrm{b}_{1}^{2}}{\alpha_{4}} f_{8}+\frac{\left(\mathrm{a}_{2}-\mathrm{b}_{2} h_{2}\right) \mathrm{d}_{1}-\left(\mathrm{b}_{1}+\mathrm{d}_{1} h_{1}\right) \mathrm{b}_{2}}{\alpha_{4}} f_{9}, \\
& f_{14}=f_{17}-\left(h_{1}+h_{2}\right) f_{19}-\frac{1}{k_{x}}\left[\frac{1}{B}+\frac{\alpha_{3}}{B \alpha_{4}}\left(\mathrm{c}_{1} \mathrm{~d}_{2}-\mathrm{c}_{2} \mathrm{~d}_{1}\right) h_{1}\right] f_{7}, \\
& f_{15}=f_{18}, \quad \text { and } f_{16}=f_{19}+\frac{\alpha_{3}}{B k_{x} \alpha_{4}}\left(c_{1} \mathrm{~d}_{2}-\mathrm{c}_{2} \mathrm{~d}_{1}\right) f_{7}
\end{aligned}
$$

$$
\begin{aligned}
& g_{10}=\left(-\frac{\alpha_{3}}{B k_{x} \alpha_{4}} \mathrm{~d}_{2}+\beta_{0} \frac{\mathrm{b}_{2}-\mathrm{d}_{2} h_{2}}{B \alpha_{4}}\right) g_{7}, \quad g_{11}=\left(\frac{\alpha_{3}}{B k_{x} \alpha_{4}} \mathrm{~d}_{1}-\beta_{0} \frac{\mathrm{b}_{1}+\mathrm{d}_{1} h_{1}}{B \alpha_{4}}\right) g_{7}, \\
& g_{12}=-\frac{\left(\mathrm{a}_{1}+\mathrm{b}_{1} h_{1}\right) \mathrm{d}_{2}-\left(\mathrm{b}_{2}-\mathrm{d}_{2} h_{2}\right) \mathrm{b}_{1}}{\alpha_{4}} g_{8}-\frac{\mathrm{a}_{2} \mathrm{~d}_{2}-\mathrm{b}_{2}^{2}}{\alpha_{4}} g_{9} \text {, } \\
& g_{13}=\frac{\mathrm{a}_{1} \mathrm{~d}_{1}-\mathrm{b}_{1}^{2}}{\alpha_{4}} g_{8}+\frac{\left(\mathrm{a}_{2}-\mathrm{b}_{2} h_{2}\right) \mathrm{d}_{1}-\left(\mathrm{b}_{1}+\mathrm{d}_{1} h_{1}\right) \mathrm{b}_{2}}{\alpha_{4}} g_{9}, \\
& g_{14}=g_{17}-\left(h_{1}+h_{2}\right) g_{19}-\left[\frac{1}{B k_{x}}+\frac{\alpha_{3}}{B k_{x} \alpha_{4}}\left(\mathrm{c}_{1} \mathrm{~d}_{2}-\mathrm{c}_{2} \mathrm{~d}_{1}\right) h_{1}+\beta_{0} \frac{\mathrm{b}_{1} \mathrm{c}_{2}-\mathrm{c}_{1} \mathrm{~b}_{2}+\mathrm{c}_{1} \mathrm{~d}_{2} h_{2}+\mathrm{c}_{2} \mathrm{~d}_{1} h_{1}}{B \alpha_{4}} h_{1}\right] g_{7} \text {, } \\
& g_{15}=g_{18}, \quad \text { and } \quad g_{16}=g_{19}+\left[\frac{\alpha_{3}}{B k_{x} \alpha_{4}}\left(\mathrm{c}_{1} \mathrm{~d}_{2}-\mathrm{c}_{2} \mathrm{~d}_{1}\right)+\beta_{0} \frac{\mathrm{b}_{1} \mathrm{c}_{2}-\mathrm{c}_{1} \mathrm{~b}_{2}+\mathrm{c}_{1} \mathrm{~d}_{2} h_{2}+\mathrm{c}_{2} \mathrm{~d}_{1} h_{1}}{B \alpha_{4}}\right] g_{7} \text {, }
\end{aligned}
$$

Furthermore, we observe that the constant terms in the shear stress expressions (18) and (32) (corresponding to Jourawski's solution for an unbroken beam) should not contribute to the Mode II energy release rate $\mathcal{G}_{\text {II }}$. Thus, the peak values of the shear interfacial stress entering Equation (44) should be computed as $\tau_{0}=\tau(0)-f_{7} / B$ and $\tau_{0}=\tau(0)-g_{7} / B$ in the balanced and unbalanced cases, respectively. As a consequence, Equations (45) and (46) should be replaced by the following ones:

$$
\mathcal{G}_{\mathrm{I}}=\frac{\mathcal{H}\left(\sigma_{0}\right)}{2 k_{z} B^{2}}\left(\sum_{i=1}^{4} f_{i}\right)^{2} \quad \text { and } \quad \mathcal{G}_{\mathrm{II}}=\frac{1}{2 k_{x} B^{2}}\left(f_{5}+f_{6}\right)^{2}
$$

and

$$
\mathcal{G}_{\mathrm{I}}=\frac{\mathcal{H}\left(\sigma_{0}\right)}{2 k_{z} B^{2}}\left(\sum_{i=1}^{6} g_{i}\right)^{2} \quad \text { and } \quad \mathcal{G}_{\mathrm{II}}=\frac{1}{2 k_{x} B^{2}}\left(k_{x} \beta_{0} \sum_{i=1}^{6} \frac{g_{i}}{\mu_{i}\left(\mu_{i}^{2}-\alpha_{3}\right)}\right)^{2} .
$$

The corrections do not affect the results and scientific conclusions of the paper. We apologize for any inconvenience caused.

Acknowledgments: We would like to thank Prof. Theodoros Loutas and Mr. Panayiotis Tsokanas of the University of Patras, Greece, for pointing out the typos.

\section{References}

1. Bennati, S.; Fisicaro, P.; Taglialegne, L.; Valvo, P.S. An Elastic Interface Model for the Delamination of Bending-Extension Coupled Laminates. Appl. Sci. 2019, 9, 3560. [CrossRef]

(C) 2020 by the authors. Licensee MDPI, Basel, Switzerland. This article is an open access article distributed under the terms and conditions of the Creative Commons Attribution (CC BY) license (http:/ / creativecommons.org/licenses/by/4.0/). 